International Journal of Medical Arts 2020; 2 [3]: 519-527.

Available online at Journal Website
https://ijma.journals.ekb.eg/
Main subject [Surgery [Obstetrics] ${ }^{*}$

Original article

\title{
Incidence of Endometriosis among Women Prepared for Laparoscopy in Unexplained Infertility and Chronic Pelvic Pain
}

\author{
Mohammed Mahmoud Mahmoud El-flahgy; Walaa Mohammed El-Bassioune; Waleed Ahmed Ayad
}

Department of Obstetrics and Gynecology, Damietta Faculty of Medicine, Al-Azhar University, Egypt

Corresponding author

Mohammed Mahmoud Mahmoud El-flahgy

Email: elflahgymohammed@yahoo.com

Received at: January 01, 2020; Revised at: June 03, 2020; Accepted at: June 03, 2020; Available online at: June 03, 2020

DOl: 10.21608/ijma.2020.22163.1080

\section{ABSTRACT}

Background: Unexplained infertility continues to be a health challenge irrespective of revolution in medical care. Endometriosis could be associated with infertility. However, its prevalence is underestimated, as it need laparoscopy for definite diagnosis.

Aim of the work: To estimate the incidence of typical and atypical [subtle] pelvic endometriosis among women with unexplained infertility and chronic pelvic pain.

Patients and Methods: A total of 100 patients with unexplained infertility [50 patients] and chronic pelvic pain [50 patients] who underwent diagnostic laparoscopy had been included in the current study. All were assessed clinically after full history taking and underwent ultrasound, then prepared for laparoscopy. The main outcome was laparoscopic and histopathologic diagnosis of pelvic endometriosis, and the association between endometriosis and different patient characteristics and other risk factors had been analyzed.

Results: Endometriosis was diagnosed by laparoscopy in nearly 33 of patients included in this study of which 29 cases [12 with unexplained infertility and 17 with chronic pelvic pain] were confirmed by histopathologic examination. Thus, the final incidence of endometriosis was $29 \%$. Development of endometriosis was significantly associated with positive family history, dysmenorrhea and higher CA125.

Conclusion: Pelvic endometriosis is a frequent association with unexplained infertility and chronic pelvic pain. It should be considered in those women particularly when there was positive family history, dysmenorrhea or higher CA125.

Keywords: Chronic pelvic pain; Endometriosis; Laparoscopy; Unexplained infertility; CA125.

This is an open access article under the Creative Commons license [CC BY] [https://creativecommons.org/licenses/by/2.0/]

Please cite this article as: El-flahgy MM, El-Bassioune WM, Ayad WA. Incidence of Endometriosis among Women Prepared for Laparoscopy in Unexplained Infertility and Chronic Pelvic Pain. IJMA 2020; 2[3]: 519-527.

* Main subject and any subcategories have been classified according to research topic. 


\section{INTRODUCTION}

Endometriosis describes the existence of endometrial like glands and stroma away from cavity of the uterus. Clinical manifestations are due to repeated bleeding into the surrounding tissues with subsequent inflammatory reactions and scar formation with adhesions. Endometriosis can be chronic and progressive in a subset of patients [1].

Certain females are prone to endometriosis, who had specific predisposing factors, such as female age, genetic factors, environmental factors, and the interactions between such factors. An early menarche is also positively associated with endometriosis, and disease runs in families as discovered in twin and family studies ${ }^{[2]}$.

The prevalence of endometriosis is underestimated because of the need for laparoscopy, which is considered the gold standard, to confirm the diagnosis. At least $10 \%$ of all females in reproductive age are prone to the disease [3]. In females and teenage girls who had chronic pelvic pain, about $50 \%-60 \%$ and up to $50 \%$ of infertile females develop endometriosis. In addition, postmenopausal women with hormonal replacement therapy complained from endometriosis ${ }^{[4]}$.

The link between infertility and endometriosis had been reported in previous the literature. However, cause-and-effect relationship has not yet determined. In endometriosis, many mechanisms seem to be responsible for infertility. It includes ovulatory dysfunction due to altered folliculogensis, impaired quality of oocytes, luteal phase defects, abnormal embryogenesis and reduced fertilization power. In addition, abnormal peritoneal fluid, ectopic endometrial and immunological aberrations are proposed[5].

Tomassetti and D'Hooghe[6] evaluating the association between endometriosis and infertility, and reported that, endometriosis is an etiology of infertility. They reach this by the strong association between endometriosis and infertility in domains of their prevalence, temporal relation, intrauterine insemination and assisted reproductive techniques, and the favorable outcome of infertility treatment after removal of the endometriosis lesions

Biomarkers are searched for early diagnosis of endometriosis and its associated ovarian cancer. However, there is no consensus about non-invasive reliable biomarker. Considerable efforts were exerted to discover less invasive biomarker for confirm diagnosis of endometriosis. Cancer antigen 125 [CA-125] was found to be elevated in endometriosis [especially in stages III and IV, and with existence of endometriomas]. However, the evidence regarding its role is still limited [7].

At laparoscopy, endometriosis appearance is markedly variable. Sometimes, it appears as small lesions or implantations in peritoneal and/or the ovarian structures. These implants appear as subtle red or white lesions, clear "bubble" lesions, small hemorrhagic cysts, or white fibrotic lesions. In addition, endometriosis could appear as large ovarian endometriomas ${ }^{[3]}$.

Many Gynecologists underestimate the magnitude of this problem due to misbelieve that endometriosis is a rare disease. The diagnosis of endometriosis increased when atypical lesions were considered in the diagnostic criteria ${ }^{[8]}$.

\section{AIM OF THE WORK}

The aim of the current work was to estimate the incidence of pelvic endometriosis in cases of chronic pelvic pain and infertile women [the conditions which is not yet investigated well] attending Al- Azhar University hospital and planned to do diagnostic laparoscopy.

\section{PATIENTS AND METHODS}

This is an observational clinical study that was conducted at department of "Obstetrics and Gynecology", Al-Azhar University Hospital [New Damietta] during the period from the $1^{\text {st }}$ of April 2019 to the last of October 2019. It included 100 women; 50 women with chronic pelvic pain $>6$ months and not responding to traditional treatment and 50 patients with unexplained infertility. Patients aged 20- 40 years, with unexplained infertility either primary or secondary, and with chronic pelvic pain $>6$ months that did not respond to traditional medical treatment, were included in the study. Otherwise, females with reported previous endometriosis were excluded from the study.

Patients were of two groups: Group [1] included women with chronic pelvic pain $>6$ months and not responding to traditional treatment [n=50]. Group [2] included patients with unexplained infertility, [the cause of the fertility impairment cannot be 
discovered by usage of standard investigations like semen analysis, assessment for ovulation and tubal patency] [ $n=50]$.

The calculated sample size of the study was 50 participants for each group at $5 \%$ level of significance and $80 \%$ power, using the following formula: $\mathrm{N}=\left[\mathrm{Z} 1_{-\alpha / 2}+\mathrm{Z} 1_{-\beta}\right]^{2} \sigma 1^{*} \sigma 2 / \delta^{2}$, where, Z1$\alpha / 2=1.96, Z 1-\beta=0.842, \sigma=\operatorname{SD}[0.54,0.29], \delta=$ Expected difference detected between the studied groups regarding LDL/HDL ratio [0.2] [9], $\alpha=$ acceptable level of a false positive result [level of significance $=0.05$ ] and $\beta=$ acceptable level of a false negative result [0.20].

Ethical consideration: The study protocol was investigated and approved by the Medical Ethics Committees of the Al-Azhar Faculty of Medicine. In addition, after explanation of the nature of the study, each patient included in the study signed an informed consent.

All participants were submitted to full history taking [personal, menstrual, contraceptive, compliant, and sexual history], complete clinical examination [general, abdominal and local gynecologic examination], and trans-vaginal ultrasound. Ultrasound scan was done by a Voluson 730 Pro device [GE, Milan, Italy] with a multifrequency volume endovaginal probe. Diagnosis of ovarian endometrioma was considered if the typical pattern of "a circular-shaped homogeneous hypoechoic mass of low-levels echoes" was distinguished on the B-mode ultrasonography [10].

The endometrioma volume, the antral follicles count [AFC] was assessed bilaterally. The volumes of endometrioma were calculated according to the formula: volume $=$ height $\times$ length $\times$ width $\times 0.5233$ and expressed in $\mathrm{cm}^{3}$. Antral follicles count was done in the $2^{\text {nd }}$ day of menstrual cycle by $2 \mathrm{D}$ transvaginal ultrasound. All females were investigated in the dorsal lithotomy position, by a high-resolution transvaginal ultrasound [TVS] $5 \mathrm{MHz}$ probe. At first, the uterus was evaluated in the transverse and sagittal planes. Second, the ovaries were determined and their size was measured in three orthogonal planes. Ovarian cysts were diagnosed as endometriomas when they looked as well circumscribed thick-walled cysts which contained homogenous low-level internal echos ["ground glass"]. Measurements were documented from the inside of the cyst wall in three orthogonal planes. The average of the three diameters [D1 + D2 + D3] was calculated and used in statistical analysis.

The adnexa were also systematically investigated for tubal dilatation. Ovarian mobility was investigated by a combination of mild pressure with the vaginal probe and abdominal pressure with free hand as in a bimanual examination. The examination procedure was completed as described elsewhere ${ }^{[11]}$.

All data were documented in a database file [Microsoft Excel for Windows spreadsheet] to simplify data entry and manipulations.

The severity of endometriosis as graded by TVS and compared to laparoscopic data. Standard laparoscopy was done in all cases using 3 ports: umbilical, left and right iliac regions. $10 \mathrm{~mm}$ trocar \& cannula will be used for the umbilical port while $5 \mathrm{~mm}$ trocars \&cannula used for ports in the iliac regions. Equipment's used were Karl Storz ${ }^{\mathrm{TM}}$ video camera [single chip], light source [Xenon 300] with fiberoptic light cable, Karl Storz ${ }^{\mathrm{TM}}$ endoscope $[10 \mathrm{~mm}$ diameter, $0^{\circ}$ ], $\mathrm{CO}_{2}$ gas insufflator and video monitor. Instruments were $5 \mathrm{~mm}$ traumatic \& atraumatic graspers, scissors \& suction-irrigation. Coagulation was monopolar or bipolar diathermy. To ensure complete evaluation of the pelvis, inspection was done in a systematic manner in an anticlockwise fashion starting from the right adnexa and peritoneum of right side of pelvic wall [including ovarian fossa], to peritoneum of anterior abdominal wall and vesical peritoneum, then left adnexa and peritoneum of left side of pelvic wall ending with the pouch of Douglas and utero-sacral ligaments [12]. Cases with reported Endometriosis were classified one of four grades depending on its location, extent, and depth of implants; existence and gravity of adhesions; and existence and size of ovarian endometriomas according to the revised American Society for Reproductive Medicine [rASRM] classification of endometriosis [13].

Statistical analysis of data: The collected data were managed by the statistical package for social sciences [SPSS] version 19 [SPSS Inc, Chicago, USA], running on IBM compatible computer. Qualitative results were expressed in frequency and percentage, and quantitative results expressed in mean [for central tendency] and standard deviation [SD; for dispersion]. The independent samples, 
student [t] test and Chi square tests were used for comparison between quantitative and qualitative data respectively. $p$ value $<0.05$ was set as the marginal of significance.

\section{RESULTS}

The present study comprised 100 women within the reproductive age; 50 women with chronic pelvic pain $>6$ months and not responding to traditional treatment and 50 patients with unexplained infertility. The mean age was $23.32 \pm 1.6$ years, the mean parity was $1.76 \pm 0.89$ and mean $\mathrm{BMl}$ was $24.93 \pm 3.04$ $\mathrm{kg} / \mathrm{m}^{2}$. In addition, there were 29 women suffered from primary infertility, 21 suffered from secondary infertility. The mean duration of primary infertility was $3.76 \pm 1.38$ years, and of secondary infertility was $3.09 \pm 0.94$ years. In addition, the mean duration of chronic pelvic pain was $3.78 \pm 1.45$ years.

In the present study, the age and BMI were comparable between both groups. However, GroupI had significantly higher mean parity when compared to group-II [2.02 \pm 0.77 vs $1.5 \pm 0.93$ respectively]. Endometriosis was discovered in 12 patients of group I and 17 patients of group II, with no significant difference between groups [Table 1].

In the present study, there were 9 [31.03\%] women suffered from endometriosis in women with primary infertility, 3 [14.3\%] in women with secondary infertility \&17 [34.0\%] in women with chronic pelvic pain, poly cystic ovary [PCO] present in 2 women [6.9\%], 0 [0.0\%] \& 3 [6.0\%], complicated ovarian cyst present in 3 [10.3\%], 2 [9.5\%] \& 5 [10.0\%], pelvic inflammatory disease [PID] present in $4[13.8 \%], 7$ [33.3\%] \& 3 [6.0\%], pelvic adhesion present in $0[0.0 \%], 3[14.3 \%] \& 4[8.0 \%]$, pelvic congestion present in 4 [13.8\%], 3 [14.3\%] \& 6 [12.0\%], retroverted retroflexed uterus [RVF] present in 1 [3.4\%], 1 [4.8\%] \& 4 [8.0\%] and fibroid present in 1 [3.4\%], 0 [0.0\%] \& 2 [4.0\%], respectively [Table
2]. The age, parity and BMI were nearly comparable between positive and negative cases of endometriosis [age; $23.14 \pm 1.76$ years in positive cases Vs $23.39 \pm 1.57$ years in negative, parity; $1.86 \pm 0.79$ Vs $1.72 \pm 0.93$ and BMl; $24.35 \pm 5.02$ $\mathrm{kg} / \mathrm{m} 2$ Vs $25.16 \pm 1.66 \mathrm{~kg} / \mathrm{m} 2$ respectively]. There were 25 women [86.21\%] presented with family history of endometriosis in positive cases Vs 12 [16.9\%] in negative cases, 6 cases [20.69\%] presented with contraception in positive Vs 20 [28.17\%] in negative, mean age of menarche was $12.35 \pm 1.37 \mathrm{Vs} 12.61 \pm 1.49$ years and mean age of marriage was $21.09 \pm 1.74$ Vs $21.16 \pm 1.92$ years respectively. There were 16 [55.2\%] of the positive endometriosis suffered from dyspareunia Vs 28 [39.4\%] in negative women with endometriosis, 18 [62.1\%] women suffered from dysmenorrhea Vs 27 [38.03\%], Menorrhagia present in 9 [31.1\%] in positive Vs 18 [25.4\%], uterosacral nodule presents in 7 [24.1\%] in positive Vs 9 [12.7\%], nodule in Douglas pouch present in $5[17.2 \%]$ in positive Vs 9 [12.7\%] and fixed uterus present in 6 [20.7\%] Vs 7 [9.9\%], respectively. Follicle stimulating hormone $[\mathrm{FSH}]$, luteinizing hormone $[\mathrm{LH}]$ and prolactin were nearly comparable between positive and negative cases of endometriosis of the studied cases. Cancer antigen-125 [CA125] increased significantly in women with positive endometriosis when compared with negative cases [Table 3].

According to ASRM classifications, endometriosis in primary infertility was of stages 1 , 2, 3 and 4 among $33.3 \%, 33.3 \%, 22.2 \%$ and $11.1 \%$ respectively, while in secondary infertility the percentages were $33.3 \%, 33.3 \%, 33.3 \%$ and $0.0 \%$ with the same order and finally in chronic pelvic pain, it was Stages 1, 2, 3 and 4 among 35.3\%, 29.4\%, $23.6 \%$ and $7.1 \%$ respectively. There was no significant difference between groups regarding stages of endometriosis [Table 4].

Table [1]: Comparison between group I and II demographic data and incidence of endometriosis

\begin{tabular}{|l|c|c|c|c|}
\hline Parameter & Group I & Group II & Test & P-value \\
\hline Age [Years] & $23.5 \pm 1.62$ & $23.1 \pm 1.61$ & 1.36 & 0.17 \\
\hline Parity [No] & $2.02 \pm 0.77$ & $1.5 \pm 0.93$ & 3.04 & $0.003^{*}$ \\
\hline BMl [kg/m2] & $24.61 \pm 3.85$ & $25.24 \pm 1.89$ & 1.04 & 0.3 \\
\hline Endometriosis & $12[24.0 \%]$ & $17[34.0 \%]$ & 1.21 & 0.27 \\
\hline
\end{tabular}

Group I: 50 women with chronic pelvic pain $>6$ months and not responding to traditional treatment. Group Il: 50 patients with unexplained infertility. ${ }^{*}$ significant changes. 
Table [2]: Laparoscopic findings among the studied groups

\begin{tabular}{|l|c|c|c|}
\hline & $\begin{array}{c}\text { Primary infertility [n= } \\
\mathbf{2 9}]\end{array}$ & $\begin{array}{c}\text { Secondary infertility } \\
{[\mathbf{n}=21]}\end{array}$ & Chronic pelvic pain [n=50] \\
\hline No abnormality & $5[17.2 \%]$ & $2[9.5 \%]$ & $6[12.0 \%]$ \\
\hline Endometriosis & $9[31.03 \%]$ & $3[14.3 \%]$ & $17[34.0 \%]$ \\
\hline PCO & $2[6.9 \%]$ & $0[0.0 \%]$ & $3[6.0 \%]$ \\
\hline Complicated ovarian cyst & $3[10.3 \%]$ & $2[9.5 \%]$ & $5[10.0 \%]$ \\
\hline PID & $4[13.8 \%]$ & $7[33.3 \%]$ & $3[6.0 \%]$ \\
\hline Pelvic adhesions & $0[0.0 \%]$ & $3[14.3 \%]$ & $4[8.0 \%]$ \\
\hline Pelvic congestion & $4[13.8 \%]$ & $3[14.3 \%]$ & $6[12.0 \%]$ \\
\hline Fixed RVF & $1[3.4 \%]$ & $1[4.8 \%]$ & $4[8.0 \%]$ \\
\hline Fibroid & $1[3.4 \%]$ & $0[0.0 \%]$ & $2[4.0 \%]$ \\
\hline
\end{tabular}

Table [3]: Associated factors with endometriosis

\begin{tabular}{|l|c|c|c|c|}
\hline Parameter & Positive [29] & Negative [71] & Test & P-value \\
\hline Age [Years] & $23.14 \pm 1.76$ & $23.39 \pm 1.57$ & 0.71 & 0.47 \\
\hline Parity [No] & $1.86 \pm 0.79$ & $1.72 \pm 0.93$ & 0.73 & 0.46 \\
\hline BMI [kg/m2] & $24.35 \pm 5.02$ & $25.16 \pm 1.66$ & 1.22 & 0.23 \\
\hline Family history & $25[86.21 \%]$ & $12[16.9 \%]$ & 6.51 & $<0.001^{*}$ \\
\hline Contraception & $6[20.69 \%]$ & $20[28.17 \%]$ & 0.77 & 0.44 \\
\hline Age of menarche & $12.35 \pm 1.37$ & $12.61 \pm 1.49$ & 0.29 & 0.42 \\
\hline Age of marriage & $21.09 \pm 1.74$ & $21.16 \pm 1.92$ & 0.56 & 0.88 \\
\hline Dyspareunia & $16[55.2 \%]$ & $28[39.4 \%]$ & 1.4 & 0.15 \\
\hline Dysmenorrhea & $18[62.1 \%]$ & $27[38.03 \%]$ & 2.19 & $0.03^{*}$ \\
\hline Menorrhagia & $9[31.1 \%]$ & $18[25.4 \%]$ & 0.58 & 0.56 \\
\hline Uterosacral nodules & $7[24.1 \%]$ & $9[12.7 \%]$ & 1.42 & 0.16 \\
\hline Nodules in Douglas pouch & $5[17.2 \%]$ & $9[12.7 \%]$ & 0.59 & 0.55 \\
\hline Fixed uterus & $6[20.7 \%]$ & $7[9.9 \%]$ & 1.46 & 0.14 \\
\hline FSH & $6.24 \pm 0.59$ & $7.08 \pm 2.25$ & 1.96 & 0.06 \\
\hline LH & $6.7 \pm 1.5$ & $5.9 \pm 1.7$ & 1.94 & 0.06 \\
\hline Prolactin & $13.17 \pm 1.44$ & $12.8 \pm 1.9$ & 0.92 & 0.36 \\
\hline CA125 & $28.89 \pm 1.6$ & $13.76 \pm 2.4$ & 31.17 & $<0.001^{*}$ \\
\hline
\end{tabular}

Table [4]: Degree of endometriosis according to ASRM

\begin{tabular}{|c|c|c|c|c|c|}
\hline Endometriosis & $\begin{array}{c}\text { Primary infertility } \\
{[\mathrm{n}=9]}\end{array}$ & $\begin{array}{c}\text { Secondary infertility } \\
{[\mathrm{n}=3]}\end{array}$ & $\begin{array}{c}\text { Chronic Pelvic Pain } \\
{[\mathrm{n}=17]}\end{array}$ & Test & $p$ \\
\cline { 1 - 4 } Stage 1 & $3[33.3 \%]$ & $1[33.3 \%]$ & $6[35.3 \%]$ & & \\
\cline { 1 - 4 } Stage 2 & $3[33.3 \%]$ & $1[33.3 \%]$ & $5[29.4 \%]$ & \multirow{2}{*}{0.51} & 0.99 \\
\cline { 1 - 4 } Stage 3 & $2[22.2 \%]$ & $1[33.3 \%]$ & $4[23.6 \%]$ & & \\
\hline Stage 4 & $1[11.1 \%]$ & $0[0.0 \%]$ & $2[7.1 \%]$ & & \\
\hline
\end{tabular}

\section{DISUCSSION}

The current study aimed at establishment of the incidence of endometriosis among women with unexplained infertility and chronic pelvic pain. Results revealed that, there was no significant difference between studied groups regarding the age with the mean age [27 vs. 31] for positive and negative endometriosis respectively. These results coincides with Moini et al.[14] who evaluated 403 patients referred for laparoscopy due to infertility in Tehran between 2009 and 2010 and reported mean age $30.18 \pm 4.37$ and $30.85 \pm 4.53$ years for positive and negative cases. On the other hand, a study performed by Ashrafi et al.[15] in Iran reported a significant difference between infertile women with and without endometriosis [ $32.4 \pm 4.9$ vs. $31.4 \pm 5.2$ years respectively]. The current study revealed no significant association between endometriosis and BMI. This is comparable to the study done by Ashrafi et al.[15]. However, Shah et al.[16] performed a prospective trial using data collected of 116430 female nurses, reported inverse correlation between endometriosis and BMI.

We did not discover any significant association 
between endometriosis and infertility duration. Akande et al.[17] evaluated 192 infertile women and reported that the effects infertility duration and primary-type infertility were not shown to be statistically significant among females with endometriosis. On the other side, Moen et al.[18] evaluated 206 symptom free Norwegian women at the time of sterilization and concluded that prolonged infertility duration [ $>10$ years] itself may be a predisposing factor for endometriosis, when other causes were absent.

In our study, oral contraceptive pill therapy was associated with a reduced risk of endometriosis, although the difference did not grasp the statistical significance value, which agrees with Kashima et al.[19] trial. On the other side, a study carried out by Chapron et al.[20] in France, found that oral contraception [OC] usage for grave primary dysmenorrhea is accompanied by increased surgical diagnosis of endometriosis.

In our study, family history was prevalent among patients with endometriosis. Nouri et al.[21] determined the magnitude of endometriosis among first, second and third-degree relatives of 80 females with surgically-confirmed diagnosis, and 60 females without endometriosis at the medical university of Vienna. These data confirmed a familial tendency toward endometriosis, and proposed that, genetic risk factor could play a role in the pathogenesis of endometriosis.

Early menarche [before the age 11 years] was reported to increase the risk of endometriosis development[22], but the current study did not report any significant relation between endometriosis and age of menarche, that come in agreement with Peterson et al.[23] who evaluated 495 patients undergoing laparoscopy in San Francisco and reported that, no significant relationship exist between endometriosis and menstrual cycle characteristics, including average length, number of cycles and age at menarche.

In our study, females complained from dysmenorrhea were more prone to have endometriosis rather than females with no pain during their menstruation. So, dysmenorrhea is often considered as a diagnostic aid for endometriosis, and it considered the most commonly reported symptom. This is comparable to the study performed by Calhaz et al.[24] who evaluated 1079 Portuguese infertile women [488 of these women found to have endometriosis]. In this study, 776 women [72\%] complained of dysmenorrhea. The prevalence of low grade [minimal to mild] endometriosis and high grade [moderate to severe] endometriosis in females with dysmenorrhea were 35 and $14 \%$ respectively. In females without dysmenorrhea, these values were slightly reduced to 28 and $8 \%$, respectively.

In the present study, no statistical significant association of signs like restricted uterine mobility and adnexal tenderness with the disease was reported. On the other hand, a study performed by Khawaja et al.[25] at Aga Khan university hospital in Pakistan reported significant association between stages of endometriosis and restricted uterine mobility. They concluded that although none of clinical manifestations are confirmatory of endometriosis, positive clinical manifestations such as tenderness, reduced mobility or fixation of the uterus, retroverted uterus or a pelvic mass should always increase the suspicion index towards endometriosis.

In the current study, abnormal laparoscopic findings have been detected in $88 \%$ of patients whereas $12 \%$ had normal examination. The laparoscopic diagnosis of endometriosis is reported in 12 patients with unexplained infertility and 17 patients with chronic pelvic pain. Ozkan et al. [26] found a prevalence of $25-40 \%$ of endometriosis in infertile females.

Various cause of chronic pelvic pain had been recognized in many studies. In the current one, endometriosis was the most common etiology of chronic pelvic pain followed by pelvic congestion, pelvic inflammatory disease [PID], complicated ovarian cyst, adhesions and fibroid. In agreement of our study, Iftikar. [27] evaluated 30 patients selected for laparoscopy duo to chronic pelvic pain at Baqai Medical University and showed that $56.6 \%$ of patients had endometriosis followed by adhesion in $16.6 \%$, and $6.6 \%$ of benign cysts of the ovaries.

On the other hand, Chhetri et al.[28] included 55 females with chronic pelvic pain, laparoscopy detected pathology among 45 [81.8\%] females. This trial showed that pelvic adhesions were the commonest cause of chronic pelvic pain [present in $29 \%$ of females], followed by PID [12.7\%], endometriosis [9.1\%], pelvic congestion [7.2\%], tuberculosis [7.2\%], fibroid uterus [7.2\%], ovarian 
cysts [7.2\%] and parafimbrial cyst [3.4\%7]. In a trial performed by Memon et al. [29], the frequent pathological findings observed were tuberculosis [26.98\%], PID [20.63\%], endometriosis [14.28\%], pelvic adhesions [9.52\%] and benign ovarian cysts [7.93\%]. The high incidence of tuberculosis may be explained by wide spread of the disease in Pakistan where the study was done.

According to the WHO global tuberculosis control 2009, Pakistan ranks $8^{\text {th }}$ on the list of 22 high tuberculosis burden countries The diagnosis of peritoneal tuberculosis could be a demanding task for even an experienced physician because of nonspecific [30]. Sharma et al. ${ }^{[31]}$ evaluated 110 patients with chronic pelvic pain attending the University hospital of and detected pelvic pathology in $86.36 \%$ of patients. They reported that the most common laparoscopic finding was adhesions [40\%], followed by endometriosis [18\%], and pelvic congestion [20\%]. Hebbar and Chawla [32] revealed that the commonest pelvic pathology discovered by laparoscopic investigation was pelvic adhesions [20.9\%], then pelvic congestion [18.6\%]. Another observation was the reduced incidence of endometriosis in this part of Nepal [4.7\%]. This could be due to early child bearing, extended breast feeding and higher use of contraception.

According to ASRM classification 13[40\%] cases have minimal endometriosis, $6[18 \%]$ cases with mild endometriosis, 7 [21\%] with moderate and 7 [21\%] with severe endometriosis. on the other hand, $a$ study by Mishra et al. [33] conducted at the Institute of Kidney disease and Research Centre, Ahmedabad, Gujarat demonstrated that grade-1 endometriosis was confirmed among 119 females [66.1\%]; grade-II in 39 females [21.66\%]; grade-III in 11 females [6.11\%]; and grade-IV in 11 females [6.1\%].

Biopsy was taken from laparoscopically diagnosed females and confirmed diagnosis of endometriosis among 21 [63.6\%] females. In a study performed by Mettler et al. [34] at the University of Kiel, biopsy was taken from presumed endometriotic lesions in 164 patients and histologic reports confirmed the diagnosis in 138 [84.1\%] patients. Therefore, thorough histopathological examination should be a routine constituent in laparoscopic diagnosis and treatment of suspected endometriosis. Combination of laparoscopic and histopathologic diagnosis could be accomplished by appropriate documentation of the endometriotic lesions.

Although laparoscopy is the gold-standard diagnostic tool for endometriosis, many females who submitted to laparoscopy yielded negative results. Moreover, the possible risks and costs due to laparoscopy motivate the search for a noninvasive diagnostic technique to reduce the number of unnecessary laparoscopies. Laboratory findings showed marked variance regarding CA125 between positive and negative cases which could be considered a good Non-invasive test for diagnosing endometriosis. In agreement with our study, a metaanalysis performed by Airong et al. [35] found that the serum CA125 concentrations were associated with endometriosis, both in early and advanced grades of the disease.

In summary, laparoscopy with histological examination of excised lesions continues to be the gold standard for diagnosis of endometriosis, and individualization of treatment is crucial. The management of infertile couples should balance the efficacy, cost, safety, and risks of different treatment modalities. It is very important to confirm the diagnosis of endometriosis and select the best treatment suitable for the case whether medial or surgical.

Although sample size is justified, one limiting step is the small numbers included in each grade of endometriosis. Thus, future studies in large number of females with endometriosis are warranted.

\section{Financial and Non-Financial Relationships and Activities of Interest}

None

\section{REFERENCES}

1. Parasar P, Ozcan P, Terry KL. Endometriosis: Epidemiology, Diagnosis and Clinical Management. Curr Obstet Gynecol Rep. 2017;6[1]:34-41. [DOI: 10.1007/ s13669-017-0187-1].

2. Klemmt PAB, Starzinski-Powitz A. Molecular and Cellular Pathogenesis of Endometriosis. Curr Womens Health Rev. 2018 Jun;14[2]:106-116. [DOI: 10.2174/ 15734048 13666170306163448].

3. Gad MS, Abdel-Gayed AM, Dawoud RM, Amer AF. Prevalence of endometriosis in unexplained infertility and chronic pelvic pain in women attending Menoufia University Hospital. Menoufia Med J 2017; 30:356-60. [DOI: 10.4103/mmj.mmj_415_16]. 
4. Giudice LC. Clinical Practice-Endometriosis. N Engl J Med. 2010 Jun 24; 362[25]:2389-98. [DOI: 10.1056/ NEJMcp1000274].

5. Minici F, Tiberi F, Tropea A, Orlando M, Gangale MF, Romani F, et al. Endometriosis and human infertility: a new investigation into the role of eutopic endometrium. Hum Reprod 2008; 23, 530-7. [DOI:10.1093/ humrep/dem399].

6. Tomassetti C, D'Hooghe T. Endometriosis and infertility: Insights into the causal link and management strategies. Best Pract Res Clin Obstet Gynaecol. 2018 Aug; 51:2533. [DOI: 10.1016/j.bpobgyn.2018.06.002].

7. Coutinho LM, Ferreira MC, Rocha ALL, Carneiro MM, Reis FM. New biomarkers in endometriosis. Adv Clin Chem. 2019; 89:59-77. [DOI: 10.1016/ bs.acc. 2018.12.002].

8. Dun EC, Kho KA, Morozov VV, Kearney S, Zurawin JL, Nezhat CH. [2015]: Endometriosis in adolescents. JSLS. 2015 Apr-Jun;19[2]. pii: e2015.00019

9. Melo AS, Rosa-e-Silva JC, Rosa-e-Silva AC, Poli-Neto OB, Ferriani RA, Vieira CS. Unfavorable lipid profile in women with endometriosis. Fertil Steril. 2010 May 1;93[7]: 2433-6. [DOI: 10.1016/j.fertnstert.2009.08.043].

10. Guerriero S, Ajossa S, Mais V, Risalvato A, Lai MP, Melis GB. The diagnosis of endometriomas using colour Doppler energy imaging. Hum Reprod. 1998; 13[6]:16915. [DOI: 10.1093/ humrep/13.6.1691].

11. Van Holsbeke C, Van Calster B, Guerriero S, Savelli L, Paladini D, Lissoni AA, et al. Endometriomas: their ultrasound characteristics. Ultrasound Obstet Gynecol. 2010 Jun; 35[6]:730-40. [DOI:10.1002/uog.7668].

12. Ruan YQ, Liang WG, Huang SH. Analysis of laparoscopy on endometriosis patients with high expression of CA125. Eur Rev Med Pharmacol Sci. 2015 Apr;19[8]:1334-7. [PMID: 25967705].

13. Adamson GD. Endometriosis classification: an update. Curr Opin Obstet Gynecol. 2011 Aug; 23[4]: 213-20. [DOI: 10.1097/GCO.0b013e328348a3ba].

14. Moini A, Malekzadeh F, Kashfi F, Akhoond MR, Saei M, Mirbolok MH. Risk factors associated with endometriosis among infertile Iranian women. Arch Med Sci 2013; 9:506-514. [DOI:10.5114/ aoms. 2013.35420].

15. Ashrafi M, Sadatmahalleh SJ, Akhoond MR, Talebi M. Evaluation of risk factors associated with endometriosis with infertile women. Int J Fertil Steril. 2016; 10[1]:11-2. [DOI: 10.22074/ijfs.2016.4763]

16. Shah DK, Correia KF, Vitonis AF and Misser SA. Body size and endometriosis: results from 20 years of followup within the Nurses' Health Study II prospective cohort. Hum Reprod. 2013; 28[7]:1783-1792. [DOI:10.1093/humrep/det120].
17. Akande VA, Hunt LP, Jenkins JM. Differences in time to natural conception between women with unexplained infertility and infertile women with minor endometriosis. Hum Reprod 2004; 19[1]:96-103. [DOI:10.1093/humrep/deh045].

18. Moen MH, Rees M, Brincat M, Erel T, Gambacciani M, Lambrinoudaki I, et al. European Menopause and Andropause Society. EMAS position statement: Managing the menopause in women with a past history of endometriosis. Maturitas 2010; 67:94-97. [DOI: 10.1016/j.maturitas.2010.04.018].

19. Kashima K, Ishimaru T, Okamura H, Suginami H, Ikuma K, Murakami T. Familial risk among Japanese patients with endometriosis. Int J Gynaecol Obstet. 2004; 84 [1]:61- 64. [DOI: 10.1016/s0020-7292 [03]00340-0].

20. Chapron C, Souza C, Borghese B, Christine M, Santulli P, Bijaoui G, De Ziegler D. The past use of oral contraception for treating severe primary dysmenorrhea is associated with endometriosis especially deep infiltrating endometriosis. Hum Reprod. 2011; 26[8]:20282035 [DOI:10.1093/humrep/der156].

21. Nouri K, Ott J, Krupitz B, Huber JC, Wenzl R. Family incidence of endometriosis in first, second and thirddegree relatives: case-control study. Reprod Biol Endocrinol. 2010; 8:85. [DOI: 10.1186/1477-7827-8-85].

22. Missmer SA, Hankinson SE, Barbieri RL, Malspeis S, Willett WC. Reproductive history and endometriosis among premenopausal women. Obstet Gynecol 2004; 104:965-74. [DOI: 10.1097/01.AOG.0000142714. 54857.f8].

23. Peterson CM, Johnstone EB, Hammoud AO, Stanford JB, Varner MW, et al. ENDO Study Working Group. Risk factors associated with endometriosis: importance of study population for characterizing disease in the ENDO study. Am J Obstet Gynecol. 2013; 208[451]: 1-11. [DOI: 10.1016/j.ajog.2013.02.040].

24. Calhaz-Jorge C, Mol BW, Nunes J, Costa AP. Clinical predictive factors for endometriosis in infertile population. Hum Reprod. 2004; 19:2126-31. [DOI:10.1093/ humrep/deh374].

25. Khawaja U, Khawaja A, Gowani S, Shoukat S, Ejaz S, Ali F, Rizvi J, Nawaz F. Frequency of endometriosis among infertile women and association of clinical signs and symptoms with the laparoscopic staging of endometriosis. J Pak Med Assoc. 2009 Jan; 59 [1]:304. [PMID: 19213374]

26. Ozkan S, Murk W, Arici A. Endometriosis and infertility: epidemiology and evidence-based treatments. Ann N Y Acad Sci 2008; 1127:92-100. [DOI: 10.1196/annals. 1434.007].

27. Iftikhar R. Outcome of laparoscopy in chronic pelvic pain. JSP [International] 2008; 13[4]:155-158."

28. Chhetri S, Khanna S, Poonam, Sen B. Laparoscopic Evaluation of Chronic Pelvic Pain in Women. J Nepal 
Health Res Counc 2009; 7[14]:45-8. [DOI: 10.3126/jnhrc. v7i1. 2279].

29. Memon SR, Memon M R, Shaikh AA, Memon N Y. Role of Laparoscopy in the diagnosis of chronic pelvic pain. RMJ 2013; 38 [4]: 397-400.

30. Boabang P, Brunken M, Boxhorn J, Amo-Takyi Bk. [Chronic abdominal pains caused two cases reports]. Zentralbl Gynakol. J 2001; 123:599-603.

31. Sharma D, Dahiya K, Duhan N, Bansal R. Diagnostic laparoscopy in chronic pelvic pain. Arch Gynecol Obstet 2011; 283[2]:295-7. [DOI: 10.1007/s00404010-1354-z].

32. Hebbar S, Chawla C. Role of laparoscopy in evaluation of chronic pelvic pain. J Minim Access Surg. 2005;1[3]:116- 20. [DOI: 10.4103/0972-9941.18995].
33. Mishra W, Gaddagi RA, Aggarwal R Choudhary S, Sharma U, Patel U. Prevalence; Characteristic and Management of Endometriosis amongst Infertile Women: A One Year Retrospective Studies. J Clin Diagn Res. 2015 Jun; 9[6]: QC01-QC03. [DOI: 10.7860/JCDR/2015/13687.6125].

34. Mettler L, Schollmeyer T, Lehmann-Willenbrock E, Schmutzler A, Shukla D. Accuracy of laparoscopic diagnosis of endometriosis. JSLS 2003; 7:15-8. [PMID:12722993].

35. Shen A, Xu S, Ma Y, Guo H, Li C, Yang C, Zou S. Diagnostic value of serum CA125, CA19-9 and CA153 in endometriosis: A meta-analysis. J Int Med Res. 2015 Oct;43 [5]: 599-609. [DOI: 10.1177/ 0300060515583076]. 